\title{
Unicity of Meromorphic Functions Sharing Sets with Their Linear Difference Polynomials
}

\author{
Sheng Li and BaoQin Chen \\ College of Science, Guangdong Ocean University, Zhanjiang 524088, China \\ Correspondence should be addressed to BaoQin Chen; chenbaoqin_chbq@126.com
}

Received 23 January 2014; Accepted 20 March 2014; Published 13 April 2014

Academic Editor: Zhi-Bo Huang

Copyright (C) 2014 S. Li and B. Chen. This is an open access article distributed under the Creative Commons Attribution License, which permits unrestricted use, distribution, and reproduction in any medium, provided the original work is properly cited. \begin{abstract}
We mainly investigate the unicity of meromorphic functions sharing two or three sets with their linear difference polynomials and
\end{abstract} prove some results.

\section{Introduction and Main Results}

In this paper, we assume the reader is familiar with the fundamental results and the basic notations of the Nevanlinna theory of meromorphic functions (see, e.g., [1-3]). Let $f(z)$ be meromorphic in the whole plane. We use the notation $\rho(f)$ to denote the order of growth of the meromorphic function $f(z)$. In addition, we denote by $S(r, f)$ any quantity satisfying $S(r, f)=o(T(r, f))$, as $r \rightarrow \infty$ outside of a possible exceptional set of finite logarithmic measure. We say that a meromorphic function $a(z)$ is a small function of $f(z)$ provided that $T(r, a)=S(r, f)$. Let $S(f)$ be the set of all small functions of $f(z)$.

For a set $S \subset S(f)$, we define the following:

$$
\begin{aligned}
& E_{f}(S)=\bigcup_{a \in S}\{z \mid f(z)-a(z)=0, \text { counting multiplicities }\}, \\
& \bar{E}_{f}(S)=\bigcup_{a \in S}\{z \mid f(z)-a(z)=0, \text { ignoring multiplicities }\} .
\end{aligned}
$$

Let $f$ and $g$ be meromorphic functions. If $E_{f}(S)=E_{g}(S)$ and $\bar{E}_{f}(S)=\bar{E}_{g}(S)$, respectively, then we say that $f$ and $g$ share a set $S$ CM and IM, respectively.

Furthermore, let $c$ be a nonzero complex constant. We define the shift of $f(z)$ by $f(z+c)$, and define the difference operators of $f(z)$ by

$$
\begin{gathered}
\Delta_{c} f(z)=f(z+c)-f(z), \\
\Delta_{c}^{n} f(z)=\Delta_{c}^{n-1}\left(\Delta_{c} f(z)\right), \quad n \in \mathbb{N}, n \geq 2 .
\end{gathered}
$$

The unicity theory of meromorphic functions sharing sets is an important topic of the uniqueness theory. First of all, we recall the following theorem given by Li and Yang in [4].

Theorem A (see [4]). Let $m \geq 2$ and let $n>2 m+6$ with $n$ and $n-m$ having no common factors. Let $a$ and $b$ be two nonzero constants such that the equation $\omega^{n}+a \omega^{n-m}+b=0$ has no multiple roots. Let $S=\left\{\omega \mid \omega^{n}+a \omega^{n-m}+b=0\right\}$. Then, for any two nonconstant meromorphic functions $f$ and $g$, the conditions $E_{f}(S)=E_{g}(S)$ and $E_{f}(\{\infty\})=E_{g}(\{\infty\})$ imply $f=g$.

Yi and Lin considered the case $m=1$ with the condition that two meromorphic functions share three sets and got the result as follows.

Theorem B (see [5]). Let $S_{1}=\left\{\omega: \omega^{n}+a \omega^{n-1}+b=0\right\}$, $S_{2}=\{0\}$, and $S_{3}=\{\infty\}$, where $a, b$ are nonzero constants such that $\omega^{n}+a \omega^{n-1}+b=0$ has no repeated root and $n(\geq 4)$ is an integer. If, for two nonconstant meromorphic functions $f$ and $g, E_{f}\left(S_{j}\right)=E_{g}\left(S_{j}\right)$ for $j=1,2,3$, and $\Theta(\infty ; f)>0$, then $f \equiv g$.

Recently, a number of papers have focused on difference analogues of the Nevanlinna theory (see, e.g., [6-9]). In particular, there has been an increasing interest in studying the uniqueness problems related to meromorphic functions and their shifts or their difference operators (see, e.g., [10$16])$. 
In 2010, Zhang considered a meromorphic function $f(z)$ sharing sets with its shift $f(z+c)$ and proved the following result.

Theorem C (see [16]). Let $m \geq 2$ and let $n \geq 2 m+4$ with $n$ and $n-m$ having no common factors. Let $a$ and $b$ be two nonzero constants such that the equation $\omega^{n}+a \omega^{n-m}+b=0$ has no multiple roots. Let $S=\left\{\omega \mid \omega^{n}+a \omega^{n-m}+b=0\right\}$. Suppose that $f(z)$ is a nonconstant meromorphic function of finite order. Then $E_{f(z)}(S)=E_{f(z+c)}(S)$ and $E_{f(z)}(\{\infty\})=E_{f(z+c)}(\{\infty\})$ imply $f(z) \equiv f(z+c)$.

For an analogue result in difference operator, B. Chen and Z. Chen proved the following theorem in [10].

Theorem $\mathbf{D}$ (see [10]). Let $m \geq 2$ and let $n \geq 2 m+4$ with $n$ and $n-m$ having no common factors. Let $a$ and $b$ be two nonzero constants such that the equation $\omega^{n}+a \omega^{n-m}+b=0$ has no multiple roots. Let $S=\left\{\omega \mid \omega^{n}+a \omega^{n-m}+b=0\right\}$. Suppose that $f(z)$ is a nonconstant meromorphic function of finite order satisfying $E_{f(z)}(S)=E_{\Delta_{c} f}(S)$ and $E_{f(z)}(\{\infty\})=E_{\Delta_{c} f}(\{\infty\})$. If

$$
N\left(r, \frac{1}{\Delta_{c} f}\right)=T(r, f(z))+S(r, f),
$$

then $\Delta_{c} f \equiv f(z)$.

It is natural to ask what happens if the shift $f(z+c)$ or difference operator $\Delta_{c} f(z)$ is replaced by a general expression of $f(z)$, such as a linear difference polynomial of $f(z)$.

Here, a linear difference polynomial of $f(z)$ is an expression of the form

$$
L(z, f)=b_{k}(z) f\left(z+c_{k}\right)+\cdots+b_{0}(z) f\left(z+c_{0}\right),
$$

where $b_{k}(z) \not \equiv 0, b_{0}(z), \ldots, b_{k}(z)$ are small functions of $f(z), c_{0}, \ldots, c_{k}$ are complex constants, and $k$ is a nonnegative integer.

In this paper, our aim is to investigate the uniqueness problems of linear difference polynomials of $f(z)$. In particular, we primarily consider the linear difference polynomial $L(z, f)$ which satisfies one of the following conditions:

$$
\begin{gathered}
\text { (i) } b_{0}(z)+\cdots+b_{k}(z) \equiv 1, \\
\text { (ii) } b_{0}(z)+\cdots+b_{k}(z) \equiv 0, \\
N\left(r, \frac{1}{L(z, f)}\right)=T(r, f(z))+S(r, f) .
\end{gathered}
$$

Corresponding to the above question, we obtain the following results.

Theorem 1. Let $m \geq 2$ and let $n \geq 2 m+4$ with $n$ and $n-m$ having no common factors. Let $a$ and $b$ be two nonzero constants such that the equation $\omega^{n}+a \omega^{n-m}+b=0$ has no multiple roots. Let $S=\left\{\omega \mid \omega^{n}+a \omega^{n-m}+b=0\right\}$. Suppose that $f(z)$ is a nonconstant meromorphic function of finite order and $L(z, f)$ is of the form (4) satisfying the condition in (5). If $E_{f(z)}(S)=E_{L(z, f)}(S)$ and $E_{f(z)}(\{\infty\})=E_{L(z, f)}(\{\infty\})$, then $L(z, f) \equiv f(z)$.
Corollary 2. Let $n, m$, and $S$ be given as in Theorem 1. Suppose that $f(z)$ is a nonconstant meromorphic function of finite order satisfying the following:

$$
N\left(r, \frac{1}{\Delta_{c}^{k} f}\right)=T(r, f(z))+S(r, f) .
$$

If $E_{f(z)}(S)=E_{\Delta_{c}^{k} f(z)}(S)$ and $E_{f(z)}(\{\infty\})=E_{\Delta_{c}^{k} f(z)}(\{\infty\})$, then $\Delta_{c}^{k} f \equiv f(z)$

With an additional restriction on the order of growth of $f(z)$, we prove the following fact.

Theorem 3. Let $n, m$, and $S$ be given as in Theorem 1. Suppose that $f(z)$ is a nonconstant meromorphic function of finite order such that $\rho(f) \notin \mathbb{N}$. If $E_{f(z)}(S)=E_{L(z, f)}(S)$ and $E_{f(z)}(\{\infty\})=$ $E_{L(z, f)}(\{\infty\})$, then $L(z, f) \equiv f(z)$.

Remark 4. Note that, in Theorem 3, we do not assume that the linear polynomial $L(z, f)$ satisfies the condition in (5). In fact, since $\rho(f) \notin \mathbb{N}$, by (19), we can easily get $\rho\left(e^{h(z)}\right)=$ $\operatorname{deg}(h(z))<\rho(f)$, which implies $T\left(r, e^{h(z)}\right)=S(r, f)$. Then using a similar method as in the proof of Theorem 1, we can complete the proof of Theorem 3.

Now we may ask what happens if the condition $m \geq 2$ in Theorem 1 is replaced by a weaker condition containing the case $m=1$ or even $m=0$. By considering three sets, we get the following theorem.

Theorem 5. Let $n, m$ be nonnegative integers such that $n>m$. Let $a$ and $b$ be nonzero constants such that $\omega^{n}+a \omega^{n-m}+b=0$ has no multiple roots. Let $S_{1}=\left\{\omega: \omega^{n}+a \omega^{n-m}+b=0\right\} \neq \varnothing$, $S_{2}=\{\infty\}$, and $S_{3}=\{0\}$. Suppose that $f(z)$ is a nonconstant meromorphic function of finite order, $L(z, f)$ is of the form (4) satisfying the condition in (5), and $E_{f(z)}\left(S_{j}\right)=E_{L(z, f)}\left(S_{j}\right)$ for $j=1,2,3$. Then one has the following.

(i) If $m=0$, then $L(z, f) \equiv t f(z)$, where $t^{n}=1$.

(ii) If $n$ and $m$ are coprime, then $L(z, f) \equiv f(z)$.

Remark 6. Taking $m=1$ in Theorem 5, we can obtain an analogue result of Theorem B related to linear difference polynomials.

Furthermore, the following result is a corollary of Theorem 5 related to difference operators.

Corollary 7. Let $n, m$, and $S_{j}, j=1,2,3$, be given as in Theorem 5. Suppose that $f(z)$ is a nonconstant meromorphic function of finite order satisfying

$$
N\left(r, \frac{1}{f(z)}\right)=T(r, f(z))+S(r, f),
$$

and $E_{f(z)}\left(S_{j}\right)=E_{\Delta_{c}^{k} f}\left(S_{j}\right)$ for $j=1,2,3$. Then one has the following.

(i) If $m=0$, then $\Delta_{c}^{k} f \equiv t f(z)$, where $t^{n}=1$.

(ii) If $n$ and $m$ are coprime, then $\Delta_{c}^{k} f \equiv f(z)$.

Finally, we give some examples for our results. 
Examples. In the following, let $g(z)$ be an entire function with period 1 such that $\rho(g) \in(1, \infty) \backslash \mathbb{N}$ (see [17]).

(1) For the case (i) of condition (5), let $f_{1}(z)=e^{2 \pi i z}$, $f_{2}(z)=g(z) e^{2 \pi i z}, f_{3}(z)=e^{2 \pi i z} / g(z)$ and let $L\left(z, f_{j}\right)=2 f_{j}(z)-f_{j}(z+1)$. Then for $j=1,2,3$, $L\left(z, f_{j}\right)=f_{j}(z)$ and the sum of the coefficients of $L\left(z, f_{j}\right)$ is equal to 1 . These examples satisfy Theorems 1 and 5 but do not satisfy Theorem D.

(2) For the case (ii) of condition (5), let $f(z)=e^{z \log 2} g(z)$ and let $L(z, f)=\Delta f(z)=f(z+1)-f(z)$. Then $L(z, f)=\Delta f(z)=f(z)$, the sum of the coefficients of $L(z, f)$ equals 0 , and

$N\left(r, \frac{1}{\Delta f}\right)=N\left(r, \frac{1}{f}\right)=T(r, f(z))+S(r, f)$.

This example satisfies Theorems 1 and 5 and Corollaries 2 and 7.

(3) For Theorem 3, let $f(z)=e^{z \log 3} / g(z)$ and let $L(z, f)=f(z+1)-2 f(z)$. Then $L(z, f)=f(z)$ and the sum of the coefficients of $L(z, f)$ equals -1 . This example satisfies Theorem 3 but does not satisfy Theorem D and Theorems 1 and 5.

\section{Proof of Theorem 1}

We need the following lemmas for the proof of Theorem 1.

The difference analogue of the logarithmic derivative lemma was given by Halburd-Korhonen [7] and Chiang-Feng [6] independently. We recall the following lemmas.

Lemma 8 (see [7]). Let $f(z)$ be a nonconstant meromorphic function of finite order, $c \in \mathbb{C}$ and $\delta<1$. Then

$$
m\left(r, \frac{f(z+c)}{f(z)}\right)=o\left(\frac{T(r+|c|, f)}{r^{\delta}}\right)
$$

for all $r$ outside of a possible exceptional set with finite logarithmic measure.

Lemma 9 (see [8]). Let $c \in \mathbb{C}$, let $n \in \mathbb{N}$, and let $f(z)$ be a meromorphic function of finite order. Then for any small periodic function $a(z) \in S(f)$ with period $c$, consider the following:

$$
m\left(r, \frac{\Delta_{c}^{n} f}{f(z)-a(z)}\right)=S(r, f)
$$

where the exceptional set associated with $S(r, f)$ is of at most finite logarithmic measure.

Let $f(z)$ be a meromorphic function of finite order. Notice that if $L(z, f)(\not \equiv 0)$ is of the form (4) such that $b_{0}(z)+\cdots+b_{k}(z) \equiv 0$, then, for any given complex constant $a, L(z, a)=0$. This indicates that $L(z, f)=L(z, f-a)$ and hence

$$
\begin{aligned}
m\left(r, \frac{L(z, f)}{f-a}\right)= & m\left(r, \frac{L(z, f-a)}{f-a}\right) \\
\leq & \sum_{j=0}^{k} m\left(r, \frac{b_{j}(z)\left(f\left(z+c_{j}\right)-a\right)}{f-a}\right) \\
& +S(r, f)=S(r, f) .
\end{aligned}
$$

With this, one can easily prove Lemma 10 below by a similar reasoning as in the proof of the difference analogue of the second main theorem of the Nevanlinna theory in [8] by Halburd and Korhonen. We omit those details.

Lemma 10. Let $c \in \mathbb{C}$, let $f(z)$ be a meromorphic function of finite order, and let $L(z, f) \neq \equiv$ be of the form (4) such that $b_{0}(z)+\cdots+b_{k}(z) \equiv 0$. Let $q \geq 2$ and let $a_{1}, \ldots, a_{q}$ be distinct complex constants. Then

$$
\begin{aligned}
& m(r, f)+\sum_{i=1}^{q} m\left(r, \frac{1}{f-a_{i}}\right) \\
& \quad \leq 2 T(r, f)-N^{*}(r, f)+S(r, f),
\end{aligned}
$$

where

$$
N^{*}(r, f):=2 N(r, f)-N(r, L(z, f))+N\left(r, \frac{1}{L(z, f)}\right)
$$

and the exceptional set associated with $S(r, f)$ is of at most finite logarithmic measure.

Remark 11. If the linear difference polynomial $L(z, f)$ is replaced by

$$
\begin{aligned}
L^{*}(z, f)= & b_{k}(z) f(z+k c) \\
& +\cdots+b_{1}(z) f(z+c)+b_{0}(z) f(z),
\end{aligned}
$$

Lemma 10 also holds even if the distinct complex constants $a_{1}, \ldots, a_{q}$ are replaced by $a_{1}(z), \ldots, a_{q}(z)$ which are distinct meromorphic periodic functions with period $c$ such that $a_{i} \epsilon$ $S(f)$ for all $i=1, \ldots, q$.

The following is the standard Valiron-Mohon'ko theorem; (see Theorem 2.2.5 in the book of Laine [2]).

Lemma 12 (see [2]). Let $f(z)$ be a meromorphic function. Then, for all irreducible rational functions in $f$,

$$
R(z, f)=\frac{P(z, f)}{Q(z, f)}=\frac{\sum_{i=0}^{p} a_{i}(z) f^{i}}{\sum_{j=0}^{q} b_{j}(z) f^{j}},
$$

with meromorphic coefficients $a_{i}(z), b_{j}(z)$ such that

$$
\begin{array}{ll}
T\left(r, a_{i}\right)=S(r, f), & i=0, \ldots, p, \\
T\left(r, b_{j}\right)=S(r, f), & j=0, \ldots, q .
\end{array}
$$


The characteristic function of $R(z, f)$ satisfies

$$
T(r, R(z, f))=d T(r, f)+S(r, f),
$$

where $d=\max \{p, q\}$.

Proof of Theorem 1. Since $f(z)$ and $L(z, f)$ share $\infty$ CM, we see that $L(z, f) \not \equiv 0$ and $N(r, L(z, f))=N(r, f(z))$. Then by Lemma 8 , we have

$$
\begin{aligned}
T(r, L(z, f))= & m(r, L(z, f))+N(r, L(z, f)) \\
\leq & m\left(r, \frac{L(z, f)}{f(z)}\right) \\
& +m(r, f(z))+N(r, f(z)) \\
\leq & \sum_{i=0}^{k} m\left(r, \frac{f\left(z+c_{i}\right)}{f(z)}\right) \\
& +\sum_{i=0}^{k} m\left(r, b_{i}(z)\right)+T(r, f(z)) \\
\leq & T(r, f(z))+S(r, f) .
\end{aligned}
$$

Since $E_{f(z)}(S)=E_{L(z, f)}(S)$, where $S=\left\{\omega \mid \omega^{n}+a \omega^{n-m}+\right.$ $b=0\}$ and the equation $\omega^{n}+a \omega^{n-m}+b=0$ has no multiple roots, we know that $(L(z, f))^{n}+a(L(z, f))^{n-m}+b$ and $f(z)^{n}+$ $a f(z)^{n-m}+b$ share $0 \mathrm{CM}$. Then from this and the condition $E_{f(z)}(\{\infty\})=E_{L(z, f)}(\{\infty\})$, there exists a polynomial $h(z)$ such that

$$
\frac{(L(z, f))^{n}+a(L(z, f))^{n-m}+b}{f(z)^{n}+a f(z)^{n-m}+b}=e^{h(z)} .
$$

Suppose that $e^{h(z)} \not \equiv 1$. Note that $S=\left\{\omega \mid \omega^{n}+a \omega^{n-m}+\right.$ $b=0\}$ and the equation $\omega^{n}+a \omega^{n-m}+b=0$ has no multiple roots. Let $\omega_{1}, \ldots, \omega_{n}$ denote all different roots of the equation $\omega^{n}+a \omega^{n-m}+b=0$.

Next we prove that $T\left(r, e^{h(z)}\right)=S(r, f)$. We know that

$$
\begin{aligned}
L(z, f)-\omega_{i}= & b_{k}(z)\left(f\left(z+c_{k}\right)-f(z)\right) \\
& +\cdots+b_{0}(z)\left(f\left(z+c_{0}\right)-f(z)\right) \\
& +\left(b_{k}(z)+\cdots+b_{0}(z)\right) f(z)-\omega_{i}, \\
= & b_{k}(z) \Delta_{c_{k}} f+\cdots+b_{0}(z) \Delta_{c_{0}} f \\
& +\left(b_{k}(z)+\cdots+b_{0}(z)\right) f(z)-\omega_{i} .
\end{aligned}
$$

(i) If $b_{0}(z)+\cdots+b_{k}(z) \equiv 1$, we see that

$L(z, f)-\omega_{i}=b_{k}(z) \Delta_{c_{k}} f+\cdots+b_{0}(z) \Delta_{c_{0}} f+\left(f(z)-\omega_{i}\right)$.
Then we deduce from this, (19), and Lemma 9 that

$$
\begin{aligned}
T\left(r, e^{h(z)}\right)= & m\left(r, e^{h(z)}\right) \\
& =m\left(r, \frac{(L(z, f))^{n}+a(L(z, f))^{n-m}+b}{f(z)^{n}+a f(z)^{n-m}+b}\right) \\
& =m\left(r, \frac{\left(L(z, f)-\omega_{1}\right) \cdots\left(L(z, f)-\omega_{n}\right)}{\left(f(z)-\omega_{1}\right) \cdots\left(f(z)-\omega_{n}\right)}\right) \\
\leq & \sum_{i=1}^{n} m\left(r, \frac{L(z, f)-\omega_{i}}{f(z)-\omega_{i}}\right)+S(r, f) \\
\leq & \sum_{i=1}^{n} \sum_{j=0}^{k} m\left(r, \frac{\Delta_{c_{j}} f}{f(z)-\omega_{i}}\right) \\
& +\sum_{i=1}^{n} \sum_{j=0}^{k} m\left(r, b_{j}(z)\right)+S(r, f) \\
= & S(r, f) .
\end{aligned}
$$

(ii) If $b_{0}(z)+\cdots+b_{k}(z) \equiv 0$, we have

$$
L(z, f)-\omega_{i}=b_{k}(z) \Delta_{c_{k}} f+\cdots+b_{0}(z) \Delta_{c_{0}} f-\omega_{i} .
$$

From this, (19), and Lemma 9, we get

$$
\begin{aligned}
T\left(r, e^{h(z)}\right)= & m\left(r, e^{h(z)}\right) \\
\leq & \sum_{i=1}^{n} m\left(r, \frac{L(z, f)-\omega_{i}}{f(z)-\omega_{i}}\right)+S(r, f) \\
\leq & \sum_{i=1}^{n} \sum_{j=0}^{k} m\left(r, \frac{\Delta_{c_{j}} f}{f(z)-\omega_{i}}\right) \\
& +\sum_{i=1}^{n} m\left(r, \frac{1}{f(z)-\omega_{i}}\right)+S(r, f) \\
= & \sum_{i=1}^{n} m\left(r, \frac{1}{f(z)-\omega_{i}}\right)+S(r, f) .
\end{aligned}
$$

Applying Lemma 10 to $f(z)$, we get

$$
\begin{aligned}
& \sum_{i=1}^{n} m\left(r, \frac{1}{f(z)-\omega_{i}}\right) \\
& \leq 2 T(r, f(z))-m(r, f(z))-2 N(r, f(z)) \\
& \quad+N(r, L(z, f))-N\left(r, \frac{1}{L(z, f)}\right)+S(r, f) \\
& =T(r, f(z))-N\left(r, \frac{1}{L(z, f)}\right)+S(r, f) .
\end{aligned}
$$


Then the assumptions in (5), (24), and (25) yield the following:

$$
\begin{aligned}
T\left(r, e^{h(z)}\right) \leq & T(r, f(z)) \\
& -N\left(r, \frac{1}{L(z, f)}\right)+S(r, f)=S(r, f) .
\end{aligned}
$$

To sum up, we now prove that $T\left(r, e^{h(z)}\right)=S(r, f)$. Rewriting (19), we get

$$
\begin{aligned}
& (L(z, f))^{n-m}\left[(L(z, f))^{m}+a\right] \\
& \quad=\left[f(z)^{n}+a f(z)^{n-m}+b-b e^{-h(z)}\right] e^{h(z)} .
\end{aligned}
$$

Denote $F(z)=f(z)^{n}+a f(z)^{n-m}$. It follows from Lemma 12 and $m>0$ that

$$
T(r, F(z))=n T(r, f(z))+S(r, f) .
$$

Hence, $S(r, F)=S(r, f)$.

By (18) and (27) and applying the second main theorem for three small target functions, we deduce the following:

$$
\begin{aligned}
& T(r, F(z)) \\
& \leq \bar{N}(r, F(z))+\bar{N}\left(r, \frac{1}{F(z)}\right) \\
& +\bar{N}\left(r, \frac{1}{F(z)+b-b e^{-p(z)}}\right)+S(r, F) \\
& \leq \bar{N}(r, f(z))+\bar{N}\left(r, \frac{1}{f(z)^{n-m}\left[f(z)^{m}+a\right]}\right) \\
& +\bar{N}\left(r, \frac{1}{(L(z, f))^{n-m}}\right)+\bar{N}\left(r, \frac{1}{(L(z, f))^{m}+a}\right) \\
& +S(r, f) \\
& \leq \bar{N}(r, f(z))+\bar{N}\left(r, \frac{1}{f(z)}\right)+\bar{N}\left(r, \frac{1}{f(z)^{m}+a}\right) \\
& +\bar{N}\left(r, \frac{1}{L(z, f)}\right)+T\left(r, \frac{1}{(L(z, f))^{m}+a}\right) \\
& +S(r, f) \\
& \leq T(r, f(z))+T\left(r, \frac{1}{f(z)}\right)+T\left(r, \frac{1}{f(z)^{m}+a}\right) \\
& +T\left(r, \frac{1}{L(z, f)}\right)+m T(r, L(z, f))+S(r, f) \\
& \leq(m+2) T(r, f(z)) \\
& +(m+1) T(r, L(z, f))+S(r, f) \\
& \leq(2 m+3) T(r, f(z))+S(r, f) \text {. }
\end{aligned}
$$

By combining (28) and (29), we have

$$
(n-2 m-3) T(r, f(z)) \leq S(r, f),
$$

which contradicts with $n \geq 2 m+4$.

Now we turn to consider the case $e^{h(z)} \equiv 1$. Equation (19) yields the following:

$$
(L(z, f))^{n}+a(L(z, f))^{n-m} \equiv f(z)^{n}+a f(z)^{n-m} .
$$

Set $\varphi(z)=L(z, f) / f(z)$, and we have

$$
f(z)^{m}\left(\varphi(z)^{n}-1\right)=-a\left(\varphi(z)^{n-m}-1\right) .
$$

If $\varphi(z)$ is not a constant, (32) can be rewritten as

$$
\begin{aligned}
& f(z)^{m}(\varphi(z)-1)(\varphi(z)-\mu) \cdots\left(\varphi(z)-\mu^{n-1}\right) \\
& =-a(\varphi(z)-1)(\varphi(z)-\nu) \cdots\left(\varphi(z)-\nu^{n-m-1}\right),
\end{aligned}
$$

where $\mu=\cos (2 \pi / n)+i \sin (2 \pi / n)$ and $\nu=\cos (2 \pi /(n-m))+$ $i \sin (2 \pi /(n-m))$.

By the assumption that $n$ and $n-m$ have no common factors, we see that $\mu, \ldots, \mu^{n-1}, \nu, \ldots, v^{n-m-1}$ are different. Assume that $z_{0}$ is a $\mu^{j}$-point of $\varphi(z)$ of multiplicity $u_{j}>0$, where $1 \leq j \leq n-1$. Notice that

$$
-a\left(\varphi\left(z_{0}\right)-1\right)\left(\varphi\left(z_{0}\right)-v\right) \cdots\left(\varphi\left(z_{0}\right)-v^{n-m-1}\right)
$$

is a constant. Then (33) implies that $z_{0}$ is a pole of $f(z)^{m}$. Thus, $u_{j} \geq m$. This yields the following, for $1 \leq j \leq n-1$ :

$$
\begin{aligned}
m \bar{N}\left(r, \frac{1}{\varphi(z)-\mu^{j}}\right) & \leq N\left(r, \frac{1}{\varphi(z)-\mu^{j}}\right) \\
& \leq T(r, \varphi(z))+S(r, h) .
\end{aligned}
$$

Then by (35), we get

$$
\begin{aligned}
2 \geq \sum_{j=1}^{n-1} \Theta\left(\mu^{j}, \varphi(z)\right) & =\sum_{j=1}^{n-1}\left\{1-\varlimsup_{r \rightarrow \infty} \frac{\bar{N}\left(r, 1 /\left(\varphi(z)-\mu^{j}\right)\right)}{T(r, \varphi(z))}\right\} \\
& \geq \sum_{j=1}^{n-1}\left(1-\frac{1}{m}\right)=(n-1)\left(1-\frac{1}{m}\right),
\end{aligned}
$$

which is impossible with $m \geq 2$ and $n \geq 2 m+4$.

Hence, $\varphi(z)$ is a constant. Since $f(z)$ is a nonconstant meromorphic function, we deduce from (32) that $\varphi(z) \equiv 1$. This yields $L(z, f) \equiv f(z)$, which completes the proof of Theorem 1.

\section{Proof of Theorem 5}

Since $f(z)$ is a nonconstant meromorphic function of finite order, $E_{f(z)}\left(S_{j}\right)=E_{L(z, f)}\left(S_{j}\right)$ for $j=1,2,3, S_{1}=\left\{\omega: \omega^{n}+\right.$ $\left.a \omega^{n-m}+b=0\right\}, S_{2}=\{\infty\}$, and $S_{3}=\{0\}$, we have $L(z, f) \not \equiv$ $0, N(r, L(z, f))=N(r, f(z))$, and $N(r, 1 / L(z, f))=N(r$, $1 / f(z))$, and we also get (18) and (19). 
Since $f(z)$ and $L(z, f)$ share $0, \infty \mathrm{CM}$, there exists a polynomial $h^{*}(z)$ such that

$$
\frac{L(z, f)}{f(z)}=e^{h^{*}(z)} .
$$

By Lemma 8, we see that

$$
\begin{aligned}
T\left(r, e^{h^{*}(z)}\right)= & m\left(r, e^{h^{*}(z)}\right)=m\left(r, \frac{L(z, f)}{f(z)}\right) \\
\leq & \sum_{j=0}^{k} m\left(r, \frac{f\left(z+c_{j}\right)}{f(z)}\right) \\
& +\sum_{j=0}^{k} m\left(r, b_{j}(z)\right)+S(r, f) \\
= & S(r, f) .
\end{aligned}
$$

As in the proof of Theorem 1, we see that $T\left(r, e^{h(z)}\right)=$ $S(r, f)$ still holds in both cases (i) and (ii).

Rewriting (19), we have

$$
\begin{gathered}
(L(z, f))^{n}+a(L(z, f))^{n-m}-e^{h(z)} f(z)^{n} \\
-a e^{h(z)} f(z)^{n-m}=b\left(e^{h(z)}-1\right) .
\end{gathered}
$$

Combining this and (37), we get

$$
\begin{aligned}
& \left(e^{n h^{*}(z)}-e^{h(z)}\right) f(z)^{n}+a\left(e^{(n-m) h^{*}(z)}-e^{h(z)}\right) f(z)^{n-m} \\
& \quad=b\left(e^{h(z)}-1\right)
\end{aligned}
$$

Suppose that $e^{n h^{*}(z)}-e^{h(z)} \not \equiv 0$. If $m=0$, (40) becomes

$$
(a+1)\left(e^{n h^{*}(z)}-e^{h(z)}\right) f(z)^{n}=b\left(e^{h(z)}-1\right) .
$$

By the condition that $b \neq 0, S_{1}=\left\{\omega:(a+1) \omega^{n}+b=0\right\} \neq \varnothing$ implies $a \neq-1$.

It follows from (38), (41), and $T\left(r, e^{h(z)}\right)=S(r, f)$ that

$$
\begin{aligned}
n T(r, f)+S(r, f) & =T\left(r,\left(e^{n h^{*(z)}}-e^{h(z)}\right) f(z)^{n}\right) \\
& =T\left(r, b\left(e^{h(z)}-1\right)\right)=S(r, f),
\end{aligned}
$$

which is a contradiction, since $n \geq 1$. that

If $m \geq 1$, it follows from (38), (41), and $T\left(r, e^{h(z)}\right)=S(r, f)$

$$
\begin{aligned}
n T(r, f)+S(r, f)= & T\left(r,\left(e^{n h^{*}(z)}-e^{h(z)}\right) f(z)^{n}\right) \\
= & T\left(r,-a\left(e^{(n-m) h^{*}(z)}-e^{h(z)}\right) f(z)^{n-m}\right. \\
& \left.\quad+b\left(e^{h(z)}-1\right)\right) \\
\leq & (n-m) T(r, f)+S(r, f) .
\end{aligned}
$$

That is impossible.
Therefore, $e^{n h^{*}(z)}-e^{h(z)} \equiv 0$. Notice that $a, b \neq 0$. Using a similar method, we can prove that $e^{(n-m) h^{*}(z)}-e^{h(z)} \equiv 0$. Then (40) implies that $e^{h(z)} \equiv 1$.

If $m=0$, we have $e^{n h^{*}(z)} \equiv 1$. Obviously, $e^{h^{*}(z)}$ is a constant. Set $t=e^{h^{*}(z)}$. Thus, by (37), we get $L(z, f) \equiv t f(z)$, where $t^{n}=1$.

If $n$ and $m$ are coprime, $e^{n h^{*}(z)} \equiv 1$ and $e^{m h^{*}(z)} \equiv 1$ imply that $e^{h^{*}(z)} \equiv 1$. Thus, by (37), we get $L(z, f) \equiv f(z)$. Thus, Theorem 5 is proved.

\section{Conflict of Interests}

The authors declare that they have no conflict of interests.

\section{Authors' Contribution}

Both authors drafted the paper and read and approved the final paper.

\section{Acknowledgments}

The authors are grateful to the editor and referees for their valuable suggestions. This work was supported by the NNSFC (no. 11171119, 11301091), the Guangdong Natural Science Foundation (no. S2013040014347), and the Foundation for Distinguished Young Talents in Higher Education of Guangdong (no. 2013LYM_0037).

\section{References}

[1] W. K. Hayman, Meromorphic Functions, Oxford Mathematical Monographs, Clarendon Press, Oxford, UK, 1964.

[2] I. Laine, Nevanlinna Theory and Complex Differential Equations, vol. 15 of de Gruyter Studies in Mathematics, Walter de Gruyter, Berlin, Germany, 1993.

[3] C.-C. Yang and H.-X. Yi, Uniqueness Theory of Meromorphic Functions, vol. 557 of Mathematics and Its Applications, Kluwer Academic Publishers, Dordrecht, The Netherlands, 2003.

[4] P. Li and C.-C. Yang, "Some further results on the unique range sets of meromorphic functions," Kodai Mathematical Journal, vol. 18, no. 3, pp. 437-450, 1995.

[5] H.-X. Yi and W.-C. Lin, "Uniqueness theorems concerning a question of Gross," Proceedings of the Japan Academy, Series A. Mathematical Sciences, vol. 80, no. 7, pp. 136-140, 2004.

[6] Y.-M. Chiang and S.-J. Feng, "On the Nevanlinna characteristic of $f(z+\eta)$ and difference equations in the complex plane," Ramanujan Journal, vol. 16, no. 1, pp. 105-129, 2008.

[7] R. G. Halburd and R. J. Korhonen, "Difference analogue of the lemma on the logarithmic derivative with applications to difference equations," Journal of Mathematical Analysis and Applications, vol. 314, no. 2, pp. 477-487, 2006.

[8] R. G. Halburd and R. J. Korhonen, "Nevanlinna theory for the difference operator," Annales Academice Scientiarum Fennicae. Mathematica, vol. 31, no. 2, pp. 463-478, 2006.

[9] I. Laine and C.-C. Yang, "Clunie theorems for difference and q-difference polynomials," Journal of the London Mathematical Society, vol. 76, no. 3, pp. 556-566, 2007. 
[10] B. Chen and Z. Chen, "Meromorphic function sharing two sets with its difference operator," Bulletin of the Malaysian Mathematical Sciences Society. Second Series, vol. 35, no. 3, pp. 765-774, 2012.

[11] B. Chen, Z. Chen, and S. Li, "Uniqueness theorems on entire functions and their difference operators or shifts," Abstract and Applied Analysis, vol. 2012, Article ID 906893, 8 pages, 2012.

[12] Z.-X. Chen and H.-X. Yi, "On sharing values of meromorphic functions and their differences," Results in Mathematics, vol. 63, no. 1-2, pp. 557-565, 2013.

[13] J. Heittokangas, R. Korhonen, I. Laine, J. Rieppo, and J. Zhang, "Value sharing results for shifts of meromorphic functions, and sufficient conditions for periodicity," Journal of Mathematical Analysis and Applications, vol. 355, no. 1, pp. 352-363, 2009.

[14] S. Li and B. Chen, "Meromorphic functions sharing small functions with their linear difference polynomials," Advances in Difference Equations, vol. 2013, article 58, 2013.

[15] S. Li and Z. Gao, "Entire functions sharing one or two finite values CM with their shifts or difference operators," Archiv der Mathematik, vol. 97, no. 5, pp. 475-483, 2011.

[16] J. Zhang, "Value distribution and shared sets of differences of meromorphic functions," Journal of Mathematical Analysis and Applications, vol. 367, no. 2, pp. 401-408, 2010.

[17] M. Ozawa, "On the existence of prime periodic entire functions," Kōdai Mathematical Seminar Reports, vol. 29, no. 3, pp. 308-321, 1978. 


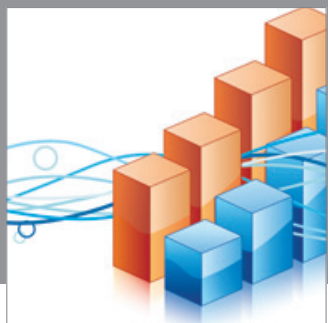

Advances in

Operations Research

mansans

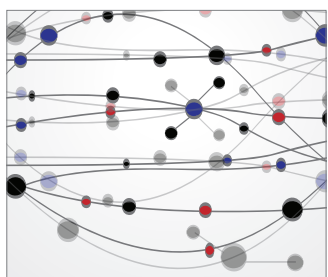

The Scientific World Journal
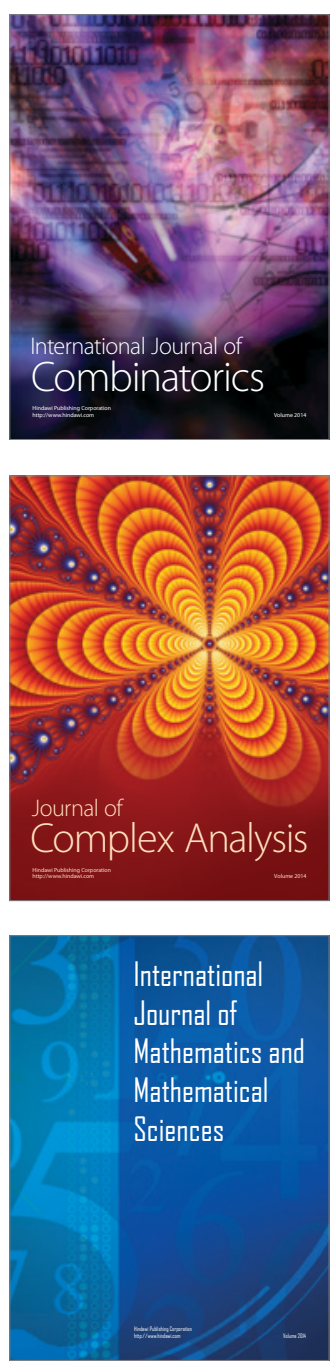
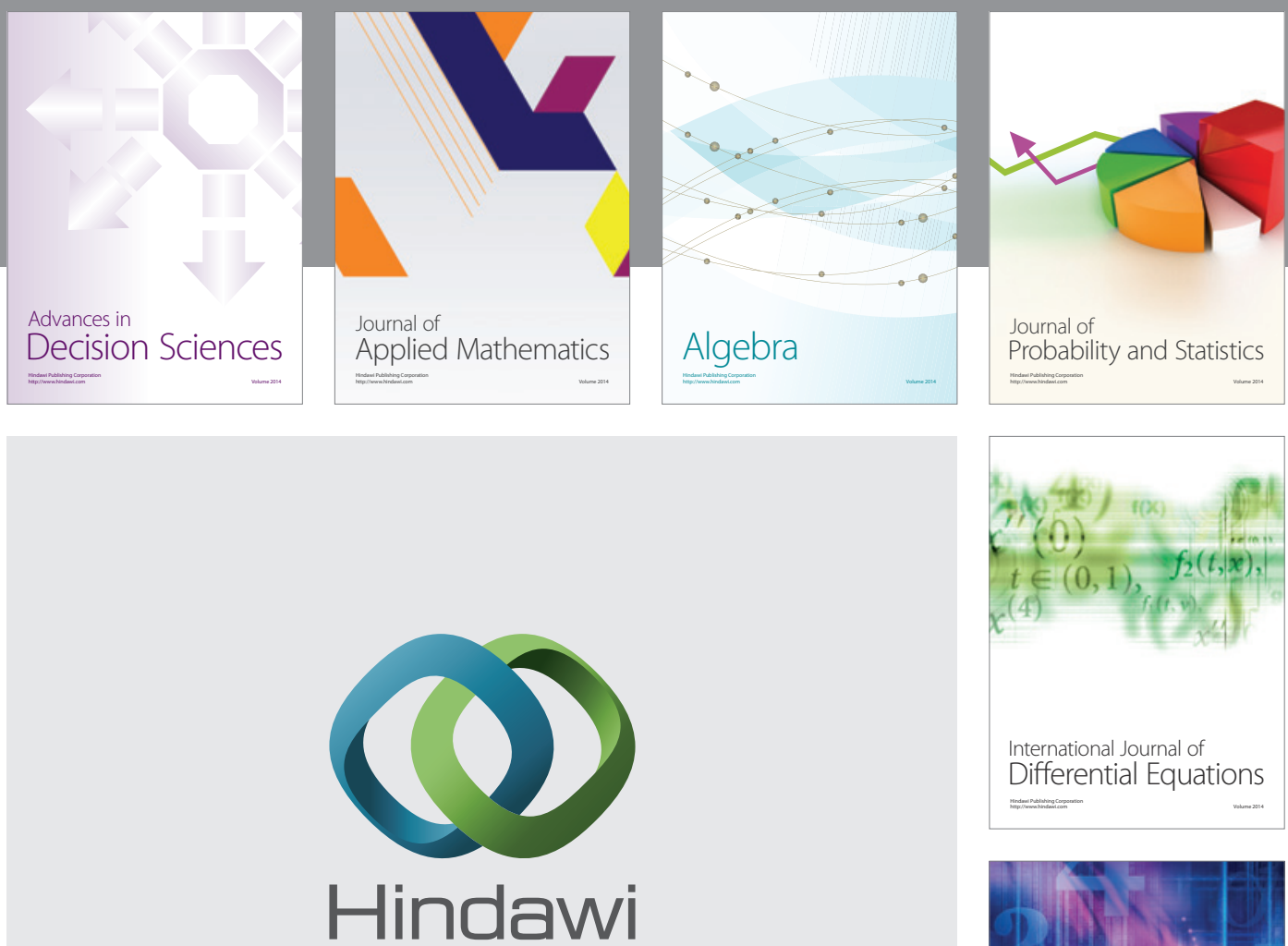

Submit your manuscripts at http://www.hindawi.com
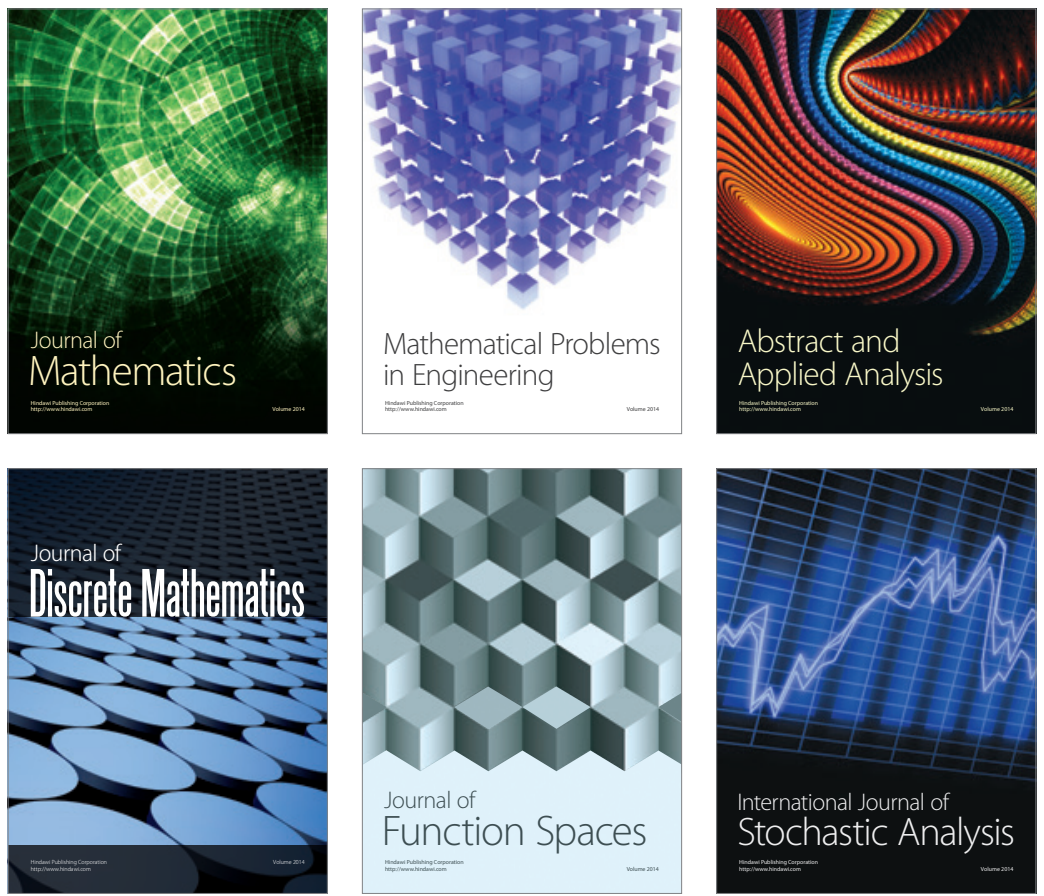

Journal of

Function Spaces

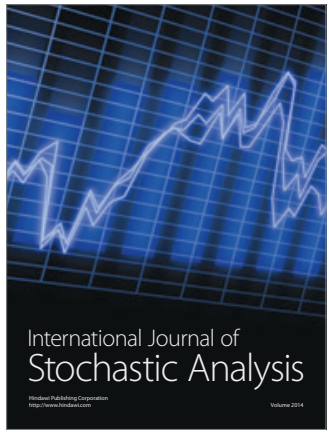

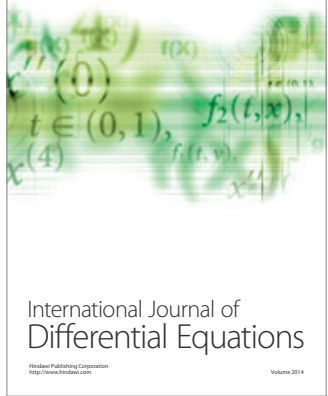
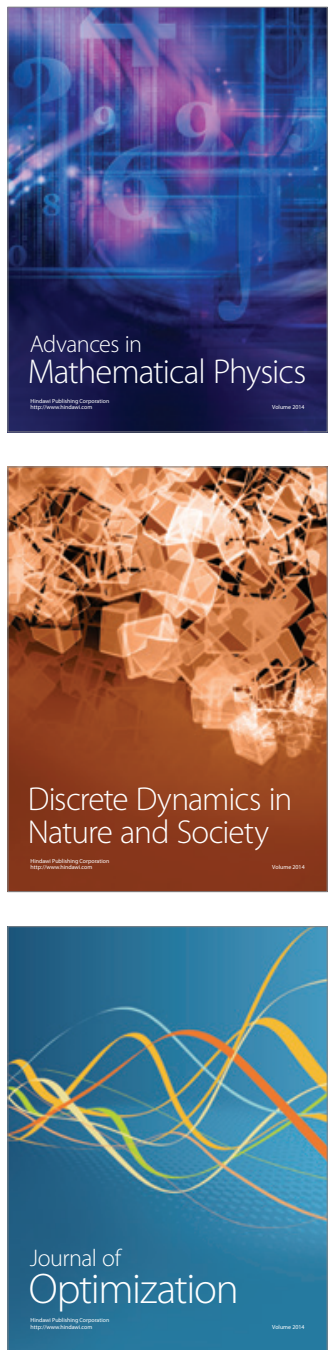